\section{Photodynamische Therapie in der Pneumologie}

F. Stanzel ${ }^{1}$, K. Häußinger ${ }^{1}$, D. Geiger ${ }^{1}$, J. Pichler $^{2}$, W. Sauer ${ }^{1}$, H. Stepp ${ }^{2}$

${ }^{1}$ Asklepios Fachkliniken München-Gauting, Pneumologische Klinik (Chefarzt: Prof. Dr. K. Häußinger)

${ }^{2}$ Laser-Forschungslabor, Urologische Klinik und Poliklinik, Klinikum Großhadern, Ludwig-Maximilians-Universität München (Direktor: Prof. Dr. A. Hofstetter)

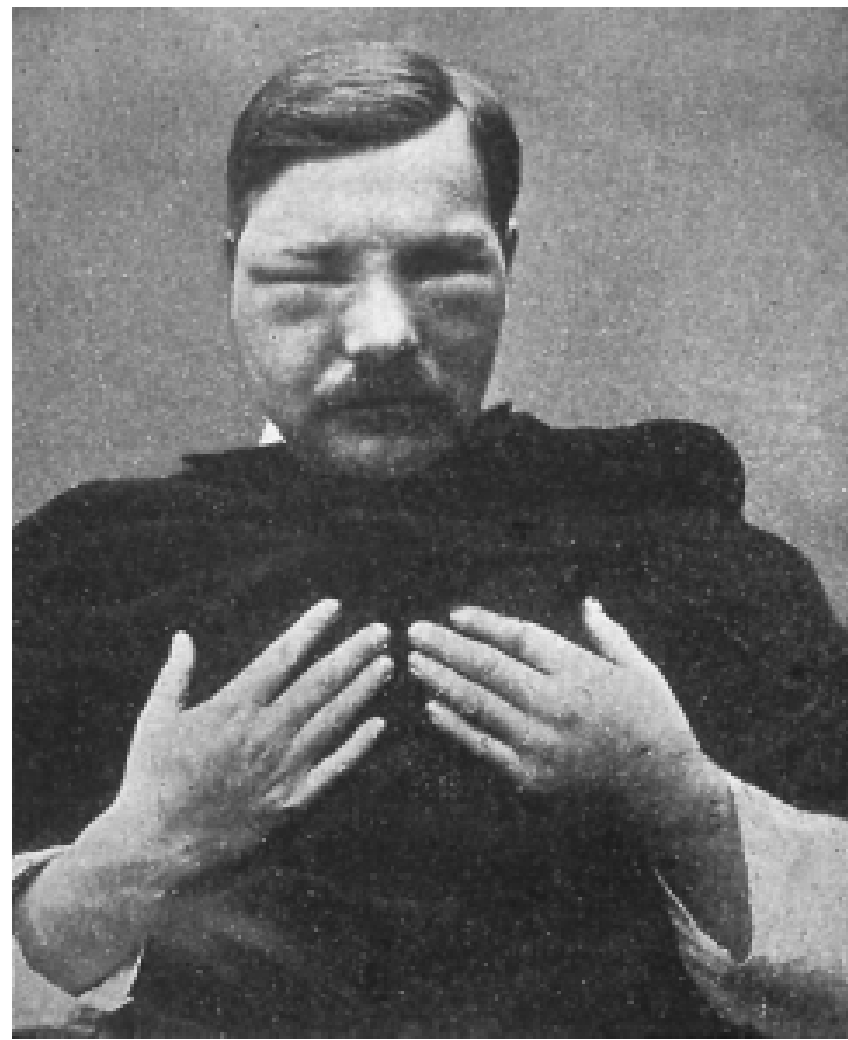

Abb. 1 Hautreaktion nach Hämatoporphyringabe und Lichtexposition im Selbstversuch 1903. legten damit den Grundstein zur heutigen Fluoreszenzdiagnostik [5,6]. Mitte der 70er Jahre wurde im Roswell Park Memorial Institut die erste erfolgreiche Behandlung maligner Tumoren tierexperimentell durchgeführt. Als Lichtquelle diente eine Xenon-Bogenlampe. Später wurden Laser mit spezifischen Wellenlängen eingesetzt. Erste Behandlungen von Frühstadien des Plattenepithelkarzinoms der Lunge beim Menschen wurden Anfang der 80er Jahre durchgeführt [2]. Die wichtigsten Beiträge zur klinischen Evaluierung dieser neuen Behandlungsmethode leisteten die Mayo Klinik, Rochester, USA, und das Tokio Medical College, Japan [7,8]. Die Zulassung von Photofrin II erfolgte in den USA und Deutschland unter anderem aufgrund einer von der FDA geprüften prospektiven randomisierten Studie der Pneumologischen Klinik Gauting Anfang 1998 [9].

Pneumologie 2000; 54: 269-277

(c) Georg Thieme Verlag Stuttgart · New York ISSN 0934-8387
Die zytotoxische Reaktion basiert auf der Aktivierung der photoaktiven Substanz durch Licht einer spezifischen Wellenlänge. Jede Lichtquelle, die Photonen mit geeigneten spektralen Eigenschaften erzeugt, kann den Sensitizer aktivieren. Laserlicht ist deshalb besonders gut geeignet, weil die konstante Wellenlänge und Kohärenz der Strahlung eine Fokussierung in eine fiberoptische Faser erleichtern. So kann das Anregungslicht durch das Fiberbronchoskop ohne wesentlichen Funktionsverlust in die Atemwege eingebracht werden [2].

In Abhängigkeit von der oberflächlichen Tumorausdehnung und der Anatomie der betroffenen Atemwege sind diverse Fasern und Strahlkörper in Gebrauch, um eine möglichst 
homogene Bestrahlung zu gewährleisten. Bei größerer Tiefenausdehnung werden die Fasern in den Tumor eingestochen, um einen Behandlungseffekt auch in tiefer gelegenen Schleimhautschichten zu erreichen [9-11].

Die Wahl der Wellenlänge hängt ab von der Absorptionscharakteristik der Substanz und der Eindringtiefe der Strahlung. Da die Eindringtiefe mit größerer Wellenlänge zunimmt, werden Substanzen bevorzugt, die in Bereichen ab $600 \mathrm{~nm}$ eine ausreichend hohe Lichtabsorption aufweisen. Licht dieser Wellenlänge dringt 3 bis $5 \mathrm{~mm}$ ins Gewebe ein und ist energiereich genug, um hier den zytotoxischen Prozess in Gang zu setzen.

Am häufigsten werden Argon-gepumpte Rhodamin-Farbstofflaser und Excimer-Laser eingesetzt. Beim Bronchialkarzinom werden Dosierungen von 200 (bis 300) mW pro cm Faserlänge bzw. pro $\mathrm{cm}^{2}$ Tumoroberfläche bzw. Energiedichten von 200 (bis 400) J pro cm bzw. $\mathrm{cm}^{2}$ angewandt. Bei zu hohen Dosierungen drohen überschießende phototoxische und unkalkulierbare thermische Effekte [2].

Nach systemischer Gabe wird der Sensitizer zunächst homogen im Gewebe verteilt, im weiteren Verlauf aber in malignen Geweben langsamer abgebaut. Dies führt zu einer selektiven Kumulation der Substanz im Tumor. Der Konzentrationsunterschied zum Normalgewebe ist am höchsten nach etwa 2 Tagen, das Verhältnis liegt bei ca. $1: 4$ [2].

Nach Aktivierung durch geeignetes Licht steht eine photooxidative Reaktion vom Typ II im Vordergrund. Dabei kommt es zu einer Energieübertragung vom Sensitizer auf Sauerstoff, es entstehen aktivierte Formen des Sauerstoffs. Wichtigstes Endprodukt dieser Kaskade ist Singlet-Sauerstoff in Zellmembranen, im Zytoplasma und in Organellen. Diese oxidative Reaktion bewirkt die Schädigung und schließlich den Tod der Zelle [12].

Der genauere Mechanismus dieser Apoptose ist komplex und basiert u.a. auf der Anreicherung von Lysylchlorin p6 in Lysosomen und von monokationischem Porphyrin in Membranen. Der Schaden an der Membran kann bereits Minuten nach Lichtexposition beobachtet werden. Er zeigt sich in Schwellung, Blasenbildung, Auslaufen von enzymbeladenen Vesikeln, Reduktion des aktiven Transports, Depolarisation der Zellwand, gesteigerter Aufnahme des Sensitizers, erhöhter Permeabilität für Chromate und in einer Inhibierung der ATPase [1].

Neben selektiv zellulären Effekten treten auch spezifische Gewebewirkungen auf. Dies sind Entzündung, Schwellung, Störung der Mikrozirkulation mit Stase, vaskulärer Kollaps, Leckage, Störung des Lymphabflusses und pH-Verschiebungen [15].

Der Effekt der PDT kann durch bestimmte Pharmaka beeinflusst bzw. modifiziert werden. Genauer untersucht ist dies für Adriamycin [16] und Glukokortikoide [17,18], die den Bereich der Tumornekrose vergrößern.

\section{Substanzen}

\section{Porfimer Sodium (Photofrin ${ }^{\circledR}$ )}

Photofrin $^{\circledR}$ (DHE oder Photofrin II) ist der am besten untersuchte Photosensitizer. Vorgängersubstanzen sind Hämatoporphyrinderivat (HpD oder Photofrin I) und Hämatoporphyrin. Photofrin II und $\mathrm{HpD}$ sind komplexe Gemische oligomerer Ester und Äther des Hämatoporphyrins. Die selektive Akkumulation und längere Verweildauer in Tumorzellen oder Tumorgewebe wurde für diese Substanzen bereits Anfang der 60 er Jahre belegt $[5,6]$.

Die Absorptionscharakteristik von Photofrin zeigt Abb. 2. Die Bestrahlung erfolgt mit $630 \mathrm{~nm}$, nutzt also wegen der besseren Eindringtiefe dieser Wellenlänge nur das kleinste Maximum. Die nekrotisierende Wirkung reicht 3 bis $5 \mathrm{~mm}$ tief. Die photosensibilisierende Wirkung der Haut erstreckt sich für diese Substanz über einen Zeitraum von 4 bis 12 Wochen [12].

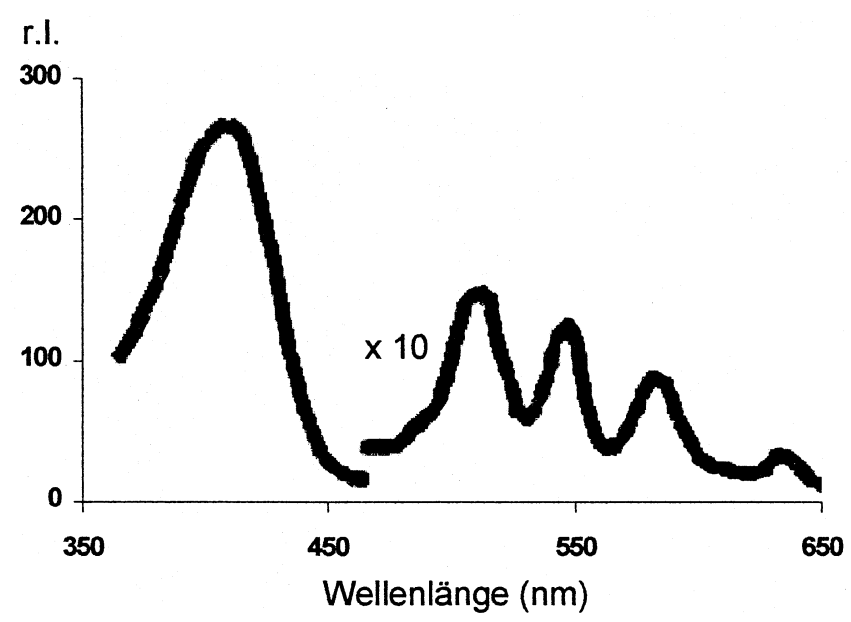

Abb. 2 Absorptionsspektrum von Photofrin.

\section{Benzoporphyrin Derivat}

Benzoporphyrinderivat (BPD) ist ein Photosensitizer der zweiten Generation. Es ist ein hydrophobes Molekül mit einem maximalen Absorptionsgradienten bei $690 \mathrm{~nm}$. Da diese Wellenlänge im Absorptionsminimum des Hämoglobins liegt, wird das Licht nicht wesentlich von Blut oder Blutkörperchen absorbiert, sondern dringt maximal in die Tiefe. Ein anderer Vorteil ist die sehr schnelle Anreicherung der Substanz im Tumor. Der optimale Behandlungszeitpunkt ist 30-150 Minuten nach intravenöser Gabe. Die Substanz wird schnell aus dem Körper eliminiert, die Photosensibilität der Haut erstreckt sich nur über wenige Tage [12].

\section{Andere Photosensitizer im klinischen Versuch}

Derzeit werden einige neue Photosensibilitatoren in klinischen Phase-II- und -III-Studien getestet. Beispiele sind Tin Etiopurpurin, SnET2 (Purlytin); Lutetium Texaphyrin (Lu-Tex); Benzoporphyrinderivat Monoacid Ring A (BPD-MA); Tetra(mhydroxyphenyl)chlorin, mTHPC (Foscan) und N-Aspartyl Chlorin e6(NPe6) [1,13]. 


\section{Delta-Aminolävulinsäure (ALA)}

Delta-Aminolävulinsäure führt im Rahmen der Hämbiosynthese in der Zelle zur Bildung von Protoporphyrin IX (PPIX). PPIX verursacht unter Lichteinfluss die Zerstörung der Zelle. ALA wird systemisch oder topisch zugeführt $[1,14]$.

Beim therapeutischen Einsatz ist der Hauptvorteil dieser Substanz die besonders kurze Hautsensibilisierung von nur 1 bis 2 Tagen. Die Bestrahlung erfolgt bei $630 \mathrm{~nm}$ mit einem Rhodamin-Farbstofflaser oder einem KTP-Laser 4-5 Stunden nach oraler Gabe von 30-40 mg/kg KG [19]. Als Nebenwirkungen werden gelegentlich Transaminasenerhöhungen beobachtet. Die lokale Reaktion ist verglichen mit der nach Photofrin ${ }^{\circledR}$ geringer ausgeprägt, der Effekt scheint auf das Epithel begrenzt zu sein [19].

Das Hauptinteresse bei Gabe dieser Substanz liegt in der Diagnostik, insbesondere im Bereich der Urologie und der Neurochirurgie. An einer kleineren Zahl von Patienten gibt es Erfahrungen mit der bronchoskopischen Diagnostik nach oraler oder inhalativer Gabe [14,19,20,21]. Autofluoreszenzverfahren erlauben aber mit höherer Zuverlässigkeit ohne Einsatz einer Substanz die Detektion maligner oder frühmaligner Veränderungen der Atemwege [22,23,24], Ultraschallverfahren in Ergänzung die Abschätzung der Tiefenausdehnung [25].

\section{Kurative Behandlung oberflächlicher endobronchialer Tumoren}

Patienten mit zentralem Bronchialkarzinom haben eine schlechte Prognose. Operation und Hochdosisstrahlentherapie sind die Standard-Therapieoptionen für Frühstadien des primären Bronchialkarzinoms bzw. beim Rezidiv. Viele dieser Patienten leiden gleichzeitig an einer chronisch obstruktiven Atemwegserkrankung oder an kardiovaskulären Erkrankungen. Die Therapiemöglichkeiten sind daher begrenzt.

Obwohl Frühkarzinome der Lunge definitionsgemäß klein sind, ist bei operativer Therapie in etwa $70 \%$ der Fälle eine Lobektomie erforderlich, in 30\% sogar eine Bilobektomie oder Pneumonektomie [26]. Oft sind Gründe, die Inoperabilität bedingen, auch Gründe, die eine Strahlentherapie ausschließen. Bei Patienten mit reduzierter pulmonaler Reserve kann die perkutane Bestrahlung die Lungenfunktion durch Pneumonitis und Fibrose weiter beeinträchtigen [27]. Aufgrund der beschränkten Lungenfunktion vieler Patienten mit Bronchialkarzinom und der Häufigkeit erneuter Primärtumoren (secundum primum) $[28,29]$ ist eine Behandlungsform, die den Verlust an Lungengewebe minimiert, besonders wünschenswert.

Für Patienten, die nicht operiert oder bestrahlt werden können, stehen nur als experimentell einzustufende Behandlungsformen wie Chemo- oder Immuntherapie oder endobronchiale lokale Behandlungsmodalitäten wie Brachytherapie, Kryotherapie, konventionelle Lasertherapie $[30,31]$ oder PDT zur Verfügung.

Von den lokalen Behandlungsmodalitäten ist neben der Brachytherapie nur die PDT systematisch untersucht und als kurativ belegt $[9,21,28,32-42]$. Ihr Vorteil gegenüber ande- ren Formen mit kurativer Therapieoption liegt in der Selektivität. Deshalb verursacht die Behandlung keinen Funktionsverlust. Die Radikalität aber wird trotz einer größeren Zahl veröffentlichter Untersuchungen kontrovers diskutiert. Das Studiendesign variiert jedoch erheblich, ein Vergleich der Ergebnisse ist daher problematisch.

Die Therapie erfolgt mit $2 \mathrm{mg} / \mathrm{kg}$ KG Photofrin ${ }^{\circledR}$ intravenös. Für die Behandlung sind ein Laser mit $630 \mathrm{~nm}$ Lichtemission, 2 Watt Leistung und spezielle Lichtleiter, die für das $\mathrm{zu}$ bestrahlende Tumorfeld geeignet sind, erforderlich (Abb.3). Die Gesamtdosis sollte $200 \mathrm{~J} / \mathrm{cm}^{2}$ betragen. Die Bestrahlungszeit errechnet sich aus der Größe des Bestrahlungsfeldes und der Gesamtlichtleistung am Austritt aus dem Applikator.
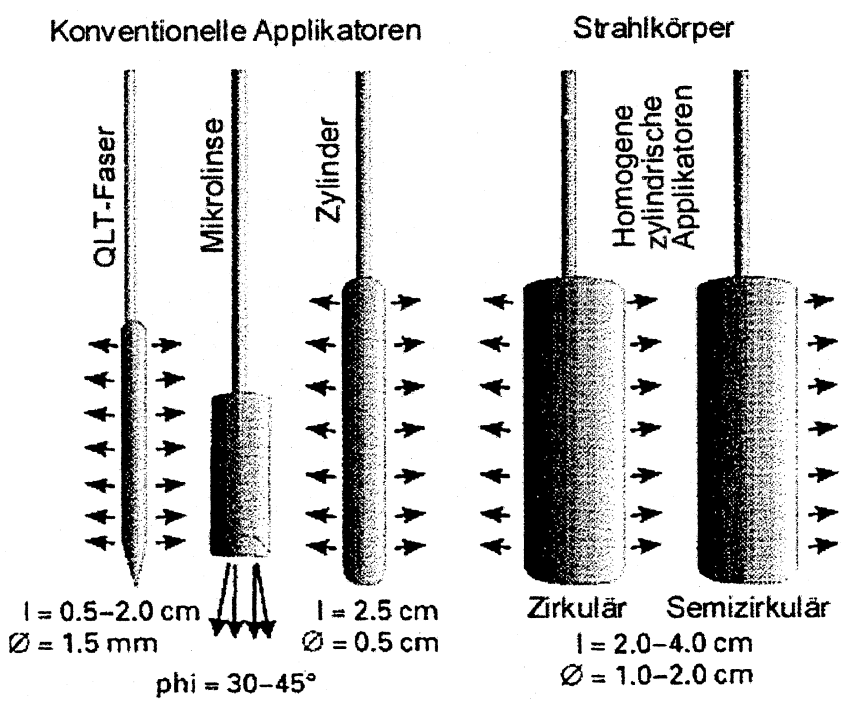

Abb. 3 Verschiedene in der kurativen PDT gebräuchliche Strahlkörper.

\section{Studien}

Phase-I- und -II-Studien von Hayata et al. in Japan [21,36] zeigen, dass nahezu $90 \%$ der oberflächlichen Tumoren unter $1 \mathrm{~cm}$ Durchmesser mit PDT komplett eradiziert werden konnten. Ähnliche Ergebnisse wurden bei Patienten mit nodulären Tumoren unter $0,5 \mathrm{~cm}$ Durchmesser erreicht. Von 81 Patienten mit kompletter Remission nach PDT starben nur 2 an der Primärerkrankung. 15 Patienten waren 5 Jahre, 3 Patienten 10 Jahre nach Behandlung noch am Leben und rezidivfrei. Die Ansprechrate nach PDT war insgesamt 71\%.

Hayata [35] fasste die Ergebnisse von mit PDT behandelten 168 Frühkarzinomen der Lunge und des Ösophagus bei 150 behandelten Patienten zusammen, getrennt in superfizielle und noduläre Wachstumsarten (Tab.1). Eine komplette Remission wurde in $95 \%$ der oberflächlichen Läsionen unter $0,5 \mathrm{~cm}$ Durchmesser und in $88 \%$ der Läsionen zwischen 0,5 und $1,0 \mathrm{~cm}$ erreicht. Die Rate kompletter Remissionen sank signifikant bei Veränderungen über $1,0 \mathrm{~cm}$ Durchmesser. Ein Rezidiv entwickelte sich bei Patienten, deren Läsionen unter $1,5 \mathrm{~cm}$ Durchmesser lagen, nur in 4 Fällen. Von den 83 nur photodynamisch behandelten Fällen mit kompletter Remis- 
Tab. 1 Ergebnis der PDT beim „Frühkarzinom“ in Abhängigkeit von Ausdehnung und Wachstumstyp

\begin{tabular}{lcl}
\hline maximale Ausdehnung & Läsionen & CR (\%) \\
\hline $\begin{array}{l}\text { oberflächlicher Typ (123 Läsionen bei 110 Patienten) } \\
<0,5 \mathrm{~cm}\end{array}$ & 64 & $61(95)$ \\
$<1,0 \mathrm{~cm}$ & 25 & $22(88)$ \\
$<2,0 \mathrm{~cm}$ & 20 & $9(45)$ \\
$>2,0 \mathrm{~cm}$ & 14 & $6(43)$ \\
nodulärer Typ (45 Läsionen bei & 43 Patienten) \\
$<0,5 \mathrm{~cm}$ & 29 & \\
$<1,0 \mathrm{~cm}$ & 9 & $27(93)$ \\
$>1,0 \mathrm{~cm}$ & 7 & $6(67)$ \\
\end{tabular}

$\mathrm{CR}=$ histologisch bestätigte komplette Remission.

Tab. 2 Tumorcharakteristik und Gründe für Inoperabilität (102 Patienten)

\begin{tabular}{lll}
\hline Tumorcharakteristik (102 Patienten) & $\mathrm{n}$ & $\%$ \\
\hline Plattenepithelkarzinom & 87 & 85 \\
Tis & 23 & 23 \\
$\mathrm{~T}_{1}$ & 63 & 62 \\
$\mathrm{~T}_{2} / \mathrm{T}_{3}$ & 8 & 8 \\
radiologisch okkult & 90 & 88 \\
Gründe für Inoperabilität & & \\
vorhergehende Lungenresektion & 48 & 47 \\
schlechte Lungenfunktion & 43 & 42 \\
multilobäre Tumoren & 20 & 20 \\
zentrale Lokalisation & 11 & 11 \\
Alter & 5 & 5 \\
Operation abgelehnt & 4 & 4 \\
mehrere Gründe & 61 & 60
\end{tabular}

Tab. 3 Ansprechraten, Überleben und Rezidivraten nach PDT in kurativer Zielsetzung ( $\mathrm{n}=100$ Patienten)

\begin{tabular}{ll}
\hline CR insgesamt & 79 Patienten (79\%) \\
\hline $95 \%$ Konfidenzintervall & $71 \%-87 \%$ \\
medianes Überleben & 3,5 Jahre \\
medianes krankheitsspezifisches Überleben & 5,7 Jahre \\
Rezidiv nach CR & 35 Patienten $(44 \%)$ \\
mediane rezidivfreie Zeit & 2,8 Jahre \\
Zeitspanne & $0,1-10,1$ Jahre \\
\hline
\end{tabular}

$\mathrm{CR}=$ histologisch bestätigte komplette Remission

sion starben 3 an der malignen Erkrankung und 47 Patienten überlebten. Von diesen waren 10 Patienten 5 Jahre und länger tumorfrei.

Bei Veränderungen vom nodulären Typ wurde eine Vollremission in $93 \%$ bei einer Größe unter 0,5 cm Durchmesser und in $67 \%$ bei 0,5 bis $1,0 \mathrm{~cm}$ Durchmesser erreicht. In den 32 Fällen

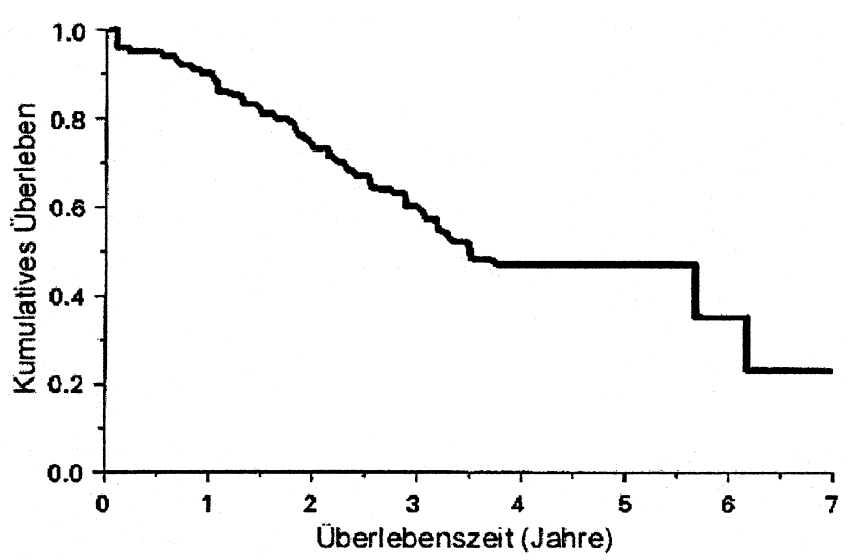

Abb. 4 Überlebenszeit nach PDT beim oberflächlichen Frühkarzinom.

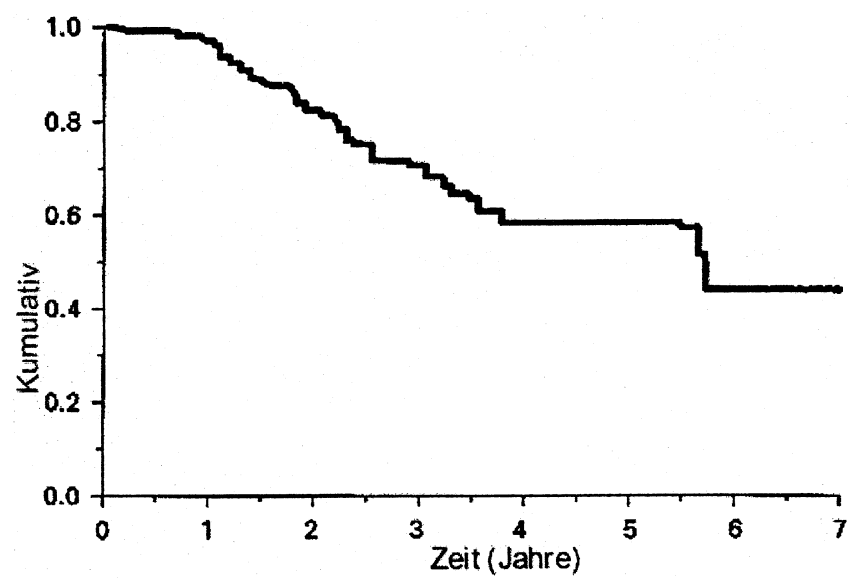

Abb. 5 Krankheitsspezifisches Überleben nach PDT beim Frühkarzinom.

mit kompletter Remission starben 8 an nicht tumorbedingten Erkrankungen, 23 überlebten. Von diesen 23 Patienten überlebten 12 Patienten fünf Jahre und länger tumorfrei.

3 offen ausgewiesene Einarmstudien zur Bewertung der Sicherheit und Effektivität der PDT mit Photofrin wurden in Deutschland, Frankreich, den Niederlanden und Kanada durchgeführt. Ihre Ergebnisse führten zur Anerkennung des Einsatzes von Photofrin ${ }^{\circledR}$ für oberflächliche Frühkarzinome der Lunge bei Patienten, die nicht für Operation oder Strahlentherapie geeignet sind [43]. Die Auswertung umfasste insgesamt 102 Patienten. Die Indikationen waren Carcinoma in situ, mikroinvasive Tumoren, die bei histologischer Beurteilung nicht den Knorpel infiltrierten und Bronchialkarzinome bei inoperablen Patienten.

Patientendaten, Tumorcharakteristika und Gründe für Inoperabilität zeigt Tab. 2 [43]. In Tab. 5 sind die Gesamtergebnisse dargestellt [42]. Die Behandlung führte bei $79 \%$ der Patienten zu einer histologisch gesicherten Vollremission (95\% Konfidenzintervall, 71-85\%). Die mittlere Überlebenszeit lag bei 3,5 Jahren (Abb. 4), das mittlere krankheitsspezifische Überleben bei 5,7 Jahren (Abb. 5) [43]. 


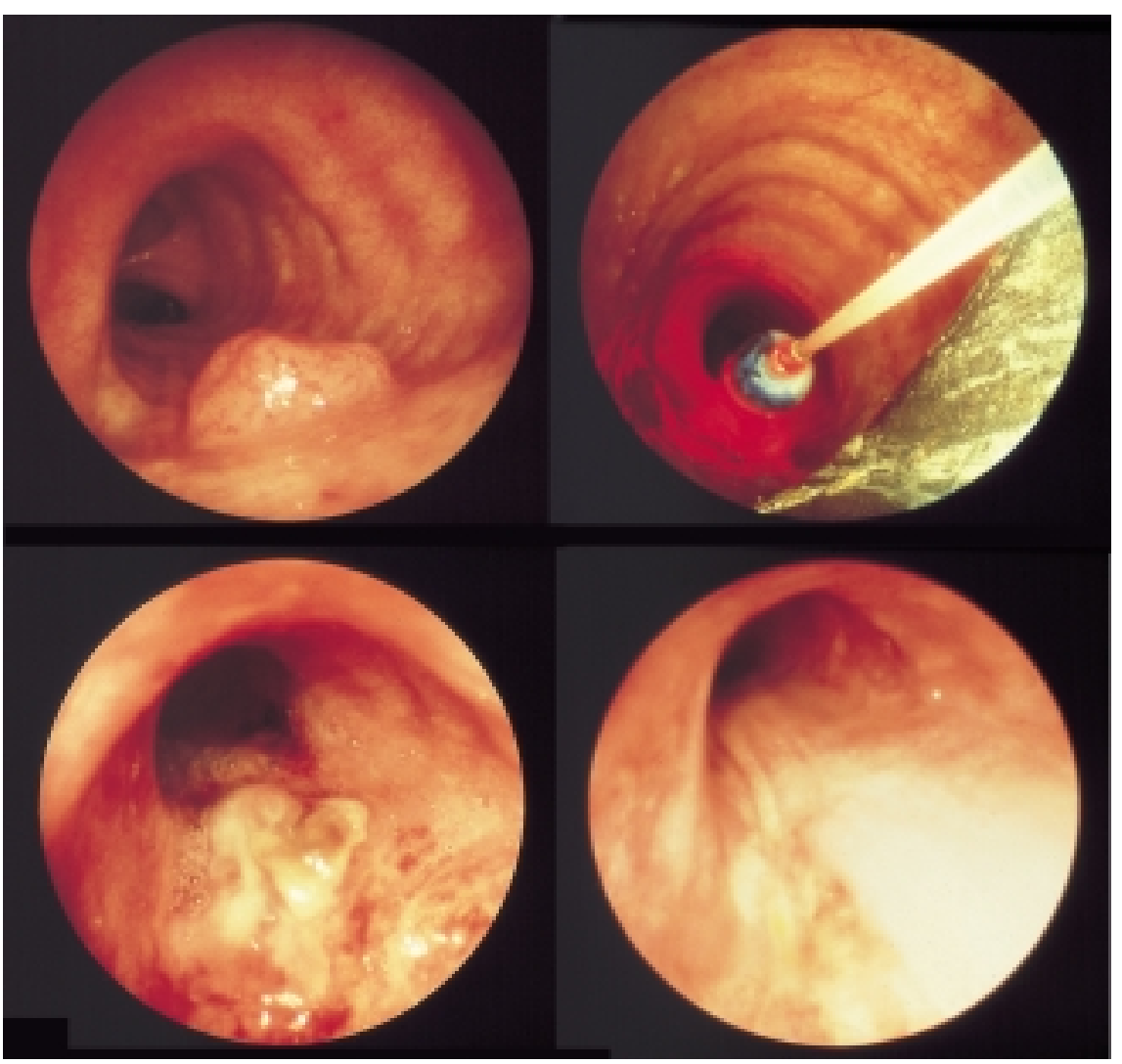

Abb. 6

a Plattenepithelkarzinom im linken Hauptbronchus

b Photodynamische Bestrahlung mit einem Feststrahlkörper

c Tumornekrose 6 Tage nach Bestrahlung d Narbenareal nach mehr als 3 Jahren.

Tab. 4 PDT beim radiologisch okkulten Bronchialkarzinom: Studien

\begin{tabular}{llll}
\hline Literatur & Läsionen & $\begin{array}{l}\text { Ansprechen } \\
\text { CR (\%) }\end{array}$ & $\begin{array}{l}\text { Überleben } \\
\text { Monate }\end{array}$ \\
\hline $\begin{array}{l}\text { Monnier et al., 1990 [51] } \\
\text { Okunaka et al., 1991 [52] }\end{array}$ & 27 & 69 & $\begin{array}{l}3-60 \\
\text { mittleres } \\
\text { Überleben 38 }\end{array}$ \\
Edell und Cortese, 1992 [18] & 14 & 71 & $7-49$ \\
Furuse et al., 1993 [20] & 59 & 83 & $14-32$ \\
Imamura et al., 1994 [33] & 39 & 64 & $4-169$ \\
Sutedja et al., 1994 [63] & 39 & 72 & $2-95$ \\
\hline
\end{tabular}

$\mathrm{CR}=$ histologisch bestätigte komplette Remission

Tab. 5 Ansprechraten der PDT gegenüber Nd:YAG-Laser-Therapie: USStudie, Europäische Studie

\begin{tabular}{lllll}
\hline & US-Studie & & \multicolumn{2}{c}{ europäische Studie } \\
& $\begin{array}{l}\text { PDT } \\
\mathrm{n}=33\end{array}$ & $\begin{array}{l}\text { Nd:YAG } \\
\mathrm{n}=37\end{array}$ & $\begin{array}{l}\text { PDT } \\
\mathrm{n}=69\end{array}$ & $\begin{array}{l}\mathrm{Nd}: \mathrm{YAG} \\
\mathrm{n}=72\end{array}$ \\
\hline CR + PR Woche 1 & $45 \%$ & $51 \%$ & $65 \%$ & $61 \%$ \\
CR + PR Monat 1 & $42 \%$ & $19 \%$ & $61 \%$ & $36 \%$ \\
\hline
\end{tabular}

$\mathrm{CR}=$ complete response: Kein Nachweis eines endoskopisch sichtbaren Tumors; $P R=$ partial response: Zunahme von $>50 \%$ vom Ausgangswert des kleinsten Lumendurchmessers oder Rückgang um $>50 \%$ der endobronchialen Verlegung.
In Tab. 4 sind die Ergebnisse der PDT oberflächlicher endobronchialer Karzinome mehrerer Studien zusammengefasst, die in den letzten 10 Jahren mitgeteilt wurden. Die Patientenzahlen sind klein, weil diese frühen Veränderungen asymptomatisch sind und meist nur zufällig bei Bronchoskopien $[28,33,34,37,41,42]$ entdeckt werden.

In unserem Zentrum wurde die PDT zwischen April 1987 und Juli 1997 bei 39 Patienten mit 52 Karzinomen (47 invasive Tumoren, 5 Carcinomata in situ) eingesetzt. Alle Veränderungen waren einschließlich $\mathrm{CT}$ radiologisch invisibel. Die Patienten waren sämtlich technisch oder funktionell inoperabel oder lehnten eine Operation ab. Das Sofortergebnis zeigte eine histologisch bestätigte Vollremission in $92 \%$. Im Langzeitverlauf (mittlere Beobachtungszeit 25,7 Monate) blieben 64\% der Patienten tumorfrei, 36\% entwickelten ein Lokalrezidiv. Bronchoskopische Kontrollen wurden alle 6 Monate durchgeführt. Es ergab sich eine Gesamtheilungsrate von 59\% [9]. Der Therapieverlauf eines dieser Patienten ist in Abb. 6a-d dokumentiert.

Die häufigsten Nebenwirkungen sind Folgen der Photosensibilisierung, meist milde bis mäßige sonnenbrandartige Reaktionen der Haut (23\%). Andere unerwünschte Wirkungen sind lokale Folgen der Behandlung, nämlich vermehrte Schleimsekretion (23\%), lokales Ödem (18\%), Narben bis hin zu Strikturen (10\%) und Ulzerationen (9\%). Perforationen wurden nicht beobachtet. 
Der Vergleich der Daten mit dem Verlauf bei unbehandelten Patienten [44] und mit den Ergebnissen der Strahlentherapie zeigt eine Überlegenheit der PDT. Im Stadium I liegt die Vollremissionsrate für die Strahlentherapie zwischen 52 bis $70 \%$, das mittlere Überleben zwischen 48 und 72 Monaten [45 - 49]. Freitag und Macha zeigten die Möglichkeit auf, PDT mit endoluminaler Brachytherapie zu kombinieren. Durch eine PDT mit Photofrin ${ }^{\circledR}$ und Bestrahlung bei $630 \mathrm{~nm}$ konnte bei sechs von 12 voroperierten Patienten mit Rezidiven bereits im ersten Kurs eine Tumoreradizierung erzielt werden. Durch weitere PDTs und zusätzliche Brachytherapien wurde bei allen Patienten eine lokale Tumorkontrolle erreicht [50].

Von Kato u. Mitarb. [51] wurde dargestellt, dass in ausgewählten Fällen eine präoperative PDT von Vorteil sein kann: Tumoren, die vom Oberlappen- oder Zwischenbronchus ausgehen, zeigen manchmal eine oberflächliche Ausdehnung in den Hauptbronchus oder in die distale Trachea und sind deswegen nicht resezierbar oder würden eine Pneumonektomie erforderlich machen. Durch eine präoperative PDT konnten 4 von 5 Patienten mit initial nicht resezierbaren Tumoren in einen operablen Zustand übergeführt werden. Von 10 Patienten, bei denen anfangs eine Pneumonektomie erforderlich gewesen wäre, konnten 7 weniger extensiv operiert werden.

Edell und Cortese [33] untersuchten die PDT als Alternative zur Operation. In einer Pilot-Studie wurden 12 Patienten mit 14 Karzinomen primär photodynamisch behandelt. Eine komplette Eradikation nach PDT wurde bei 13 von 14 Tumoren (93\%) erreicht, in nur 3 Fällen trat 7 bis 18 Monate nach der PDT ein Lokalrezidiv auf. Insgesamt rezidivierten 10 der 13 Tumoren, die eine komplette Remission zeigten, im 5-Jahresverlauf nicht, 10 der 13 Patienten (77\%) wurden von einem chirurgischen Eingriff verschont. 3 Patienten mit Tumorpersistenz nach PDT mussten sich einer Operation unterziehen, das chirurgische Tumorstadium war schließlich $\mathrm{T}_{1} \mathrm{~N}_{0} \mathrm{M}_{0}$.

\section{Palliation des Bronchialkarzinoms}

Für den Tod von Patienten mit inoperablem Bronchialkarzinom sind in der Mehrzahl der Fälle lokale Komplikationen verantwortlich. Die Patienten sterben an Asphyxie, Hämoptoe, Pneumonie oder Empyem. Eine systematische Untersuchung zeigte, dass $58 \%$ der Patienten nach vorausgegangener Operation und $83 \%$ der inoperablen Patienten an den genannten Komplikationen versterben [52]. Berücksichtigt man, dass nur 30\% der Patienten mit Bronchialkarzinom zum Zeitpunkt der Diagnosestellung operiert werden können, ist davon auszugehen, dass bei den meisten Patienten im Verlauf der Erkrankung eine palliative Maßnahme erforderlich wird.

Der palliative Einsatz der PDT zur Beseitigung zentraler Tumorobstruktionen muss zumindest ebenso effektiv sein, wie bereits etablierte konventionelle Therapieformen. Bei der Nd:YAG-Laserbehandlung wird der Laser zur Koagulation und Vaporisation von Tumorgewebe genutzt. Die Behandlung erfolgt üblicherweise unter Vollnarkose und hat sich im klinischen Einsatz bewährt [53]. Komplikationen sind selten, schwerere Blutungen, respiratorische Insuffizienz und Rhythmusprobleme kommen in weniger als $0,5 \%$ der Fälle vor [54]. Die Laserbehandlung gilt daher als Goldstandard für die palliative Therapie der partiellen oder totalen Obstruktion zentraler Atemwege durch Tumor [54,55]. Dennoch ist auch die photodynamische Behandlung eine effektive Methode, die gegenüber konventionellen Behandlungen Vorteile bietet.

\section{Klinische Daten}

Eine kürzlich publizierte prospektive Studie analysiert über einen Zeitraum von 14 Jahren den Verlauf von 175 Patienten mit primärem nichtkleinzelligen Bronchialkarzinom, die operativ, radiotherapeutisch oder mittels PDT behandelt worden waren. Die Ergebnisse zeigen, dass das Überleben in allen 3 Behandlungsgruppen in erster Linie vom Stadium der Erkrankung abhängig war [56]. Für die PDT lag das krankheitsspezifische geschätzte 5-Jahres-Überleben bei 16 Patienten mit Stadium I bei $93 \%$. Die mittleren Überlebenszeiten für die PDT lagen bei 22,5 Monaten im Stadium II, bei 5,7 Monaten im Stadium IIIA, bei 5,5 im Stadium IIIB und bei 5,0 Monaten im Stadium IV. Beim Stadium I wurde diese nicht erreicht [56].

In einer anderen prospektiven Untersuchung wurde bei 41 Patienten die photodynamische Therapie in Kombination mit Radiotherapie mit alleiniger Strahlentherapie verglichen. Dabei zeigte sich, dass die Atemwegsobstruktion nach alleiniger Bestrahlungsbehandlung nur bei $10 \%$ komplett beseitigt wurde, während nach Kombination von PDT und Radiotherapie eine Erfolgsrate von $70 \%$ erreicht wurde. Bei $20 \%$ sprach keine der Behandlungsformen an [57].

1998 wurde in zwei prospektiven randomisierten Studien die Wirksamkeit der PDT im Vergleich zur Nd:YAG-Lasertherapie zur Beseitigung partieller Tumorobstruktionen untersucht (Tab.5) [11]. Die Studie umfasste Daten von 16 Zentren in Europa und 20 Zentren in Amerika und Kanada. Beide Therapiemodalitäten führten nach einer Woche endoskopisch zu den gleichen Ergebnissen. Nach Ablauf von 4 Wochen zeigten jedoch die PDT-Patienten in 61 bzw. $42 \%$ (Europa bzW. USA/Kanada) ein weiterhin stabiles Behandlungsergebnis, während in der Nd:YAG-Laser-Gruppe nur bei 36 bzw. $19 \%$ der Patienten ein positiver Langzeiteffekt erzielt wurde. Auch die Symptomenkontrolle in Bezug auf Husten und Dyspnoe war in der PDT-Gruppe besser als in der Nd:YAGGruppe [11]. Die Zahl „unerwünschter Reaktionen“ war in beiden Gruppen gleich groß, allerdings hatten $20 \%$ der Patienten in der PDT-Gruppe ungewöhnlich lang anhaltende und heftige Hautreaktionen, die auf eine Nichtbeachtung der Vorsichtsmaßnahmen zurückgeführt wurden [1].

Die genannten Ergebnisse wurden an der vorerst jüngsten Studie an 31 Patienten mit zentralen Atemwegsobstruktionen durch maligne Tumoren bestätigt [55]. 14 dieser Patienten erhielten eine Therapie mit Photofrin ${ }^{\circledR}, 17$ eine konventionelle Lasertherapie mit dem Nd:YAG-Laser. Der Rekanalisierungserfolg wurde mittels Kontrollbronchoskopie eine Woche bzw. 4 Wochen nach der Behandlung überprüft. Nach einer Woche lagen die Erfolgsraten in der PDT-Gruppe bei $43 \%$ und in der Nd:YAG-Laser-Gruppe bei 33\%. Die endoskopische Kontrolle nach einem Monat zeigte dagegen, dass in der PDT-Gruppe noch 38,5\% der eröffneten Bronchien frei durchgängig waren, während sich in der Nd:YAG-Gruppe das positive Erstergebnis von $53 \%$ auf $23,5 \%$ reduzierte. Auch die Überlebenszeit war im PDT-Arm signifikant länger. Die Symptomkontrolle war in beiden Armen gleich. 


\section{Schlussfolgerungen}

\section{Kurative Therapie}

Für Patienten mit zentralen Frühkarzinomen der Lunge, die aus funktionellen Gründen einer externen Strahlentherapie oder Operation nicht zugeführt werden können, eröffnet die photodynamische Therapie eine neue und wirkungsvolle Therapiemöglichkeit mit kurativem Ansatz. Verglichen mit anderen Formen der Lokalbehandlung, beispielsweise endobronchialer Brachytherapie, Kryotherapie, und Elektrotherapie und thermischen Laserkoagulation basiert die PDT auf selektiver Abtötung von Tumorzellen mit nachfolgender Nekrose der bestrahlten Tumorläsionen. Ein vergleichbarer lokaler Effekt der unselektiven Behandlungsformen wird nur in singulären Fallberichten erwähnt, Studien an größeren Kollektiven liegen nicht vor. Der kurative Effekt der PDT bei frühen oberflächlichen Karzinomen ist aber systematisch untersucht und in mehreren Phase-II- und -III-Studien dokumentiert.

Als lokales Verfahren ist die PDT zwar auf zentral gelegene Veränderungen im Sichtbereich des Endoskopes und Eindringtiefen von nur wenigen Millimetern begrenzt. Die Entwicklung und Verbreitung neuer Methoden zur Frühdiagnostik des Bronchialkarzinoms wie automatisierte Sputumzytometrie und Fluoreszenzbronchoskopie führt aber bei einer zunehmenden Zahl von Patienten zur Diagnose frühmaligner Läsionen. Da die Karzinogenese bis zur Entwicklung von Invasivität Jahre beansprucht, besteht auch zeitlich die Chance, maligne Befunde rechtzeitig zu entdecken. Es ist also zu erwarten, dass eine zunehmende Zahl von Patienten der photodynamischen Therapie zugeführt werden kann.

Einer der Gründe für das Langzeitversagen ist die hohe Inzidenz metachroner Zweittumoren bei Patienten mit Bronchialkarzinom. Daher sollten alle behandelten Patienten einer regelmäßigen bronchoskopischen Nachsorge zur Kontrolle des Lokalbefundes und zum Ausschluss metachroner Tumoren unterzogen werden. Metachrone Tumoren können ebenfalls photodynamisch behandelt werden.

Ein schwer wiegendes Problem der PDT ist die lang anhaltende Photosensibilisierung der Haut. Das in den meisten klinischen Studien benutzte photosensibilisierende Medikament ist Photofrin ${ }^{\circledR}$, dessen photosensibilisierende Wirkung bis zu 3 Monate anhalten kann. Bronchoskopische Eingriffe sind auf einen Zeitraum von 2 Wochen nach der i.v. Gabe von Photofrin ${ }^{\circledR}$ begrenzt. Ein Augen- und Hautschutz sind unerlässlich, die Patienten müssen Lichtexpositionen vermeiden, insbesondere die Exposition gegenüber Sonnenlicht für einen Zeitraum von 4-8 Wochen [58]. Es ist zu erwarten, dass mit der zweiten Generation von Photosensitizern diese Zeitspanne beträchtlich kürzer wird [13].

\section{Palliative Therapie}

Die PDT hat ihre Effektivität auch in der Palliation bewiesen. Die erste photodynamische Therapie wurde Anfang der 80er Jahre durchgeführt. Seither steigt die Zahl der Behandlungen kontinuierlich.
Die derzeit verfügbaren klinischen Daten vergleichen meist die Wirksamkeit der PDT gegenüber Nd:YAG-Lasertherapie, die seit den 70er Jahren zur Palliation eingesetzt wird und als Goldstandard gilt. Beide, PDT und Nd:YAG-Laserresektion führen zur Wiedereröffnung von Atemwegsobstruktionen, die durch endoluminale Tumoren verursacht werden. Die Nd:YAG-Laserresektion hat Vorteile bei zentralen Tumoren, welche leicht mit dem starren Bronchoskop erreicht, koaguliert und abgetragen werden können. Die photodynamische Therapie andererseits wird mit dem flexiblen Bronchoskop durchgeführt, mit dem man peripherer gelegene Tumoren erreichen kann. Hierbei bedarf es keiner sofortigen mechanischen Abtragung. Wiederholte Clean-up-Bronchoskopien sind aber einige Tage nach PDT erforderlich, um Detritus zu entfernen.

Weil die meisten Patienten mit Atemwegsobstruktionen eine Kombination aus intraluminalen, submukösen oder peribronchialen Tumoranteilen aufweisen, scheint es sinnvoll, eine Kombination aus PDT und externer Bestrahlung einzusetzen [57]. In ausgewählten Fällen kann die PDT auch zur Wiedereröffnung tumorös verschlossener Atemwege genutzt werden, um eine Operation vorzubereiten oder sogar erst möglich zu machen $[20,51]$.

Als alleiniger Behandlung bei Patienten mit fortgeschrittener Erkrankung und tracheobronchialer Obstruktion kommt der PDT nur eine begrenzte Rolle zu. Mindestens gleichberechtigt sind insbesondere die Laserresektion und Brachytherapie, die zudem weniger komplex und aufwendig sind.

Ergänzend müssen einige Nachteile der PDT beachtet werden. Sie ist nicht geeignet für Patienten mit trachealen Läsionen, Obstruktionen, die beide Hauptbronchien betreffen oder in singulären Atemwegen bei pneumonektomierten Patienten. Das gelegentlich schwere, der Therapie folgende Ödem kann die Teilverlegung verschlimmern und zum Verschluss mit Lebensgefahr führen [55]. Wenn der Tumor die Bronchialwand oder Gefäßstrukturen infiltriert, kann die PDT zu Perforation oder tödlicher Blutung führen [59].

Ein weiterer Nachteil ist, dass die PDT eine Atemwegsverlegung nicht sofort beseitigen kann. Die PDT ist damit für Patienten mit akuter respiratorischer Erschöpfung durch Tumorobstruktion nicht geeignet und dürfte sich auch wegen lang anhaltender Hautsensibilisierung bei Patienten, deren Lebenserwartung nur wenige Monate beträgt, in der klinischen Routine nicht durchsetzen.

\section{Entwicklungen, zukünftige Aspekte}

Eine vordringliche Aufgabe für die Zukunft liegt darin, neue Photosensitizer mit höherer Anreicherung der Substanz im Tumor und geringerer Photosensibilisierung der Haut zu entwickeln. Eine solche Substanz würde die klinische Wirksamkeit erhöhen und sowohl lokale als auch allgemeine Nebenwirkungen der photodynamischen Therapie verringern. Auch in technisch-apparativer Hinsicht besteht Verbesserungspotenzial. Dies betrifft zum einen die Entwicklung besserer und billigerer Lichtquellen mit spezifischen Eigenschaften. Laser sollten durch konventionelle Lichtquellen mit integrierten Filtern für die Emission variabler Wellenlängen ersetzt werden, um ein besseres Eindringen in tiefere Ge- 
websschichten und eine spezifische Lichtabsorption durch den eingesetzten Sensitizer zu erhalten. Zum zweiten sollten die Lichtleitersysteme und -applikatoren für den jeweiligen Bedarf modifiziert werden: dünne flexible Fasern sollten nur in den kleineren Bronchien benutzt werden. Die Bestrahlung zentraler Läsionen sollte mit speziellen, an die komplexe Geometrie des Bronchialbaums angepassten Applikatoren erfolgen, um die Dosisverteilung zu verbessern und eine möglichst homogene Bestrahlung der Läsionen $\mathrm{zu}$ gewährleisten. Die genannten Bemühungen könnten helfen, die Praktikabilität, die Effektivität und Sicherheit dieses neuen Behandlungsverfahrens zu erhöhen und den Einsatz der PDT im klinischen Alltag breiter zu etablieren.

Die abschließende Aufgabe liegt auf klinischer Seite. Hier muss verstärkt durch randomisierte vergleichende Studien versucht werden, die Wertigkeit der lokalen Behandlungsverfahren für die verschiedenen Indikationen zu evaluieren, miteinander zu vergleichen und ihre kurative Wirksamkeit ggf. auch durch Kombination verschiedener Verfahren zu erhöhen (Tab. 3).

\section{Literatur}

${ }^{1}$ Dougherty TJ, Gomer CJ, Henderson BW, Jori G, Kessel D, Korbelik M, Moan J, Peng Q. Photodynamic therapy. J Natl Cancer Inst 1998; 90: 889-905

2 Edell ES, Cortese DA. Photodynamic Therapy - Its Use in the Management of Bronchogenic Carcinoma. Clin Chest Med 1995; 16: $455-463$

${ }^{3}$ Raab O. Über die Wirkung fluorescierender Stoffe. Infusoria Z Biol 1900; 39: 524

${ }^{4}$ Jesoniek A, von Tappeiner H. Zur Behandlung des Hautcarcinoms mit fluorescierenden Stoffen. Muench Med Wschr 1903; 47: 2043

${ }^{5}$ Lipson RL, Baldes EJ, Olsen AM. The use of a derivative of hematoporphyrin in tumor detection. J Natl Cancer Inst 1961; 26: $1-11$

${ }^{6}$ Lipson RL, Baldes EJ. The photodynamic properties of a particular hematoporphyrin derivative. Arch Dermatol 1960; 82: 508 - 516

${ }^{7}$ Cortese DA, Edell ES, Kinsey JH. Photodynamic therapy for early stage squamous cell carcinoma of the lung. Mayo Clin Proc 1997; 72: 595-602

${ }^{8}$ Kato H, Konaka C, Kawate N, Shinohara H, Kinoshita K, Noguchi M, Ootomo S, Hayata Y. Five-year disease-free survival of a lung cancer patient treated only by photodynamic therapy. Chest 1986; 90: $768-770$

${ }^{9}$ Häußinger K, Stanzel F, Sauer W. Photodynamic therapy of inoperable patients with early stage lung cancer. Abstract. World Congress for Bronchology. June 1998. 118

${ }^{10}$ Häußinger K, Stanzel F, Nimmermann C, Huber RM, Baumgartner R. Hämatoporphyrinderivate in der Therapie: Momentane Erkenntnisse und Ausblicke für die Zukunft. Atemw Lungenkrkh 1996; 22: 295-300

11 Wieman TJ, Diaz-Jiménez JP, Moghissi K, Leroy M, McCaughan J, Spinelli P, Lang N, Diaz-Agero P, York E. The Photodynamic Therapy Lung Cancer Study Group Louisville, KY, USA/Barcelona, Spain. Photodynamic therapy (PDT) with Photofrin is effective in the palliation of obstructive endobronchial lung cancer. Results of two clinical trials (abstract). ASCO. 1998

12 Levy J. Photosensitizers in photodynamic therapy. Semin Oncol 1994; $21: 4-10$

${ }^{13}$ Kessel D. PDT: Expanding the Database. An Update on New Drugs and Mechanisms of Phototoxicity. Photodynamic News 1998; $1: 2-4$
${ }^{14}$ Baumgartner R, Huber RM, Schulz H, Stepp H, Rick K, Gamarra F, Leberig A, Roth $C$. Inhalation of 5-aminolevulinic acid: A new technique for fluorescence detection of early stage lung cancer. J Photochem Photobiol B 1996; 36: 169-174

${ }^{15}$ Oleinick NL. Apoptosis in Response to Photodynamic Therapy. Photodynamic News 1998; 1: 6-9

${ }^{16}$ Edell ES, Cortese DA. Potentiation of hemato prophyrin derivative photoherapy with adriamycin. Porphyrin photosensitization Workshop. Abstract from IPA Los Angeles. July 1986. 15

17 Cowled PA, Maekenzie L, Forbes M. Potentiation of photodynamic therapy with haematoporphyrin derivatives by glucocorticoids. Cancer Lett 1986; 29: $101-114$

${ }^{18}$ Edell ES, Cortese DA. Interaction between glucocorticosteroids and hematoporphyrin derivative phototherapy. Porphyrin photosensitization Workshop (abstract). Los Angeles: IPA, July 1986.16

${ }^{19}$ Awadh N, MacAulay C, Lam S. Detection and Treatment of Superficial Lung Cancer by Using $\delta$-Aminolevulinic Acid: A Preliminary Report. J Bronchol 1997; 4: 13-17

${ }^{20}$ Hayata Y, Kato H, Konaka C, Ono J, Takizawa N. Hematoporphyrin derivative and laser photoradiation in the treatment of lung cancer. Chest 1982; 81: 269-277

${ }^{21}$ Hayata Y. The indications of photodynamic therapy in early stage lung cancer (abstract). Chest 1993; 103: 262

${ }^{22}$ Häußinger K, Pichler J, Stanzel F, Markus A, Stepp H, MorresiHauff A, Baumgartner R. Autofluorescence Bronchoscopy: The DLight System. Aus Bollinger CT, Mathur PN (Editors) . Interventional Bronchology. Prog Respir Res. Basel: Karger, 2000. 30243-252

${ }^{23}$ Häußinger K, Stanzel F, Huber RM, Pichler J, Stepp H. Autofluorescence detection of bronchial tumors with the D-Light/AF:. Diagn Ther Endosc 1999; 5: 105-112

${ }^{24}$ Häußinger K, Stanzel F, Markus A, Bolliger CT, Pichler J. Frühdiagnostik des Bronchialkarzinoms. Technisch-endoskopische Neuentwicklungen - ein Anstoß für ein neues Screening-Konzept? Pneumologie 1999; 53: 77-82

${ }^{25}$ Becker HD, Messerschmidt E, Schindelbeck F, Ott S. Endobronchialer Ultraschall. Pneumologie 1997; 51: 620-629

${ }^{26}$ Cortese DA, Pairolero PC, Bergstrahl EJ, Woolner LB, Uhlenkopp MA, Piehler JM, Sanderson DR, Bernatz PE, Williams DE, Taylor WF, Payne WS, Fontana RS. Roentgenographically occult lung cancer. A ten-year experience. J Thorac Cardiovasc Surg 1983; 86: $373-380$

${ }^{27}$ Gross NJ. Pulmonary effects of radiation therapy. Ann Intern Med 1977; 86: 81-92

28 Okunaka T, Kato H, Konaka C, Kawate N, Bonaminio A, Yamamoto H, Ikeda N, Tolentino M, Eckhauser ML, Hayata Y. Photodynamic therapy for multiple primary bronchogenic carcinoma. Cancer 1991; 68: $253-258$

${ }^{29}$ Pairolero PC, Williams DE, Bergstrahl EJ et al. Postsurgical stage 1 bronchogenic carcinoma: Morbid implications of recurrent disease. Ann Thorac Surg 1984; 38: 331 - 338

${ }^{30}$ Prakash UBS. Options in the Bronchoscopic Therapy of Airway Neoplasms. Editorial. J Bronchol 1997; 4: 97-100

31 Sutedja G, Postmus PE. Bronchoscopic treatment of lung tumors. Lung Cancer 1994; 11: 1 - 17

32 Edell ES, Cortese DA. Bronchoscopic phototherapy with hematoporphyrin derivative for treatment of localized bronchogenic carcinoma: A 5-year experience. Mayo Clin Proc 1987; 62: 8-14

33 Edell ES, Cortese DA. Photodynamic therapy in the management of early superficial squamous cell carcinoma as an alternative to surgical resection. Chest 1992; 102: 1319-1322

${ }^{34}$ Furuse K, Fukoka M, Kato H et al. A prospective phase II study on photodynamic therapy with Photofrin for centrally located early stage lung cancer. J Clin Oncol 1993; 11: 1852-1857

${ }^{35}$ Hayata Y, Kato H, Furuse K, Kusunoki Y, Suzuki S, Nimura S. Photodynamic therapy of 168 early stage cancers of the lung and 
oesophagus: A japanese multicenter study. Lasers Med Sci 1996: 11: $255-259$

${ }^{36}$ Hayata Y, Kato H, Konaka C. Photodynamic therapy (PDT) in early stage lung cancer. Lung Cancer 1993; 9: 287-294

${ }^{37}$ Imamura S, Kusunoki Y, Takifuji N et al. Photodynamic therapy and/or external beam radiation therapy for roentgenologically occult lung cancer. Cancer 1994; 73: 1608 - 1614

${ }^{38}$ Konaka C, Kato H, Havata Y. Lung cancer treated by photodynamic therapy alone: Survival for more than three years. Lasers Med Sci 1987; 2: $17-19$

${ }^{39}$ McCaughan JS, Hawley PhC, Bethel BH, Walker J. Photodynamic therapy of endobronchial malignancies. Cancer 1988; 62: 691 701

${ }^{40}$ McCaughan JS, Williams TE. Photodynamic therapy for endobronchial malignant disease: A prospective fourteen-year study. J Thorac Cardiovasc Surg 1997; 114: 940 - 947

${ }^{41}$ Monnier PH, Savary M, Fontollet Ch, Wagnieres G, Chatelain A, Comaz P, Depeursinge $\mathrm{Ch}$, van den Bergh H. Photodetection and photodynamic therapy of "early" squamous cell carcinomas of the pharynx, oesophagus and tracheobronchial tree. Lasers Med Sci 1990; 5: 149-169

${ }^{42}$ Sutedja G, Lam S, LeRiche C, Postmus PE. Response and pattern of failure after photodynamic therapy for intraluminal stage I lung cancer. J Bronchol 1994; 1: 295 - 298

${ }^{43}$ Lam S, Häußinger K, Leroy M, Sutedja T, Huber RM. Photodynamic therapy (PDT) with Photofrin, a treatment with curative potential for early stage superficial lung cancer (abstract). ASCO, 1998

${ }^{44}$ Sobue T, Suzuki T, Matsuda M, Kuroishi T, Ikeda S, Naruke T. Survival for clinical stage I lung cancer not surgically treated. Cancer 1992; 69: 685-692

${ }^{45}$ Dosoretz DE, Katin MJ, Blitzer PH, Rubenstein JH, Salenius S, Rashid M, Dosani RA, Mestas G, Siegel AD, Chadha TT. Radiation therapy in the management of medically inoperable carcinoma of the lung: Results and implications for future treatment strategies. Int J Radiat Oncol Biol Phys 1992; 24: 3-9

${ }^{46}$ Haffty BG, Goldberg MB, Gerstley J, Fischer DB, Peschel R-E. Results of radical radiation therapy in clinical stage I, technically operable non-small cell lung cancer. Int J Radiat Oncol Biol Phys 1988; 15: 69-73

${ }^{47}$ Ono R, Egawa S, Suemasu K, Sakusa M, Kitogawa T. Radiotherapy for inoperable stage I lung cancer. Jpn J Clin Oncol 1991; 21: $125-128$

${ }^{48}$ Sandler HM, Curran WJ, Turrisi AT. The influence of tumor size and treatment staging on outcome following radiation therapy alone for stage I non small cell lung cancer. Int J Radiat Oncol Bio Phys 1990; 19: 9-13

${ }^{49}$ Slotman BJ, Njo KH, Karim AB. Curative radiotherapy for technically operable stage I non-small cell lung cancer. Int J Radiat Oncol Biol Phys 1994; 29: 33 - 37

${ }^{50}$ Freitag L, Korupp A, Itzigehl I, Dankwart F, Tekolf E, Reichle G, Kullmann HJ. Erfahrungen mit Fluoreszenzdiagnostik und photodynamischer Therapie im multimodalen Therapiekonzept des operierten, rezidivierenden Bronchialkarzinoms. Pneumologie 1996; 50: 693-699

${ }^{51}$ Kato H, Konaka C, Ono J, Kawate N, Nishimiya K, Shinohara H, Saito M, Sakai H, Noguchi M, Kito T. Preoperative laser photodynamic therapy in combiantion with operation in lung cancer. J Thorac Cardiovasc Surg 1985; 90: 420-429

52 Carroll M, Morgan SA, Yamold JR, Hill JM, Wright NM. Prospective evaluation of a watch policy in patients with inoperable non-small cancer. Eur J Cancer Clin Oncol 1986; 22: 1352-1356

${ }^{53}$ Bölcskei PL, Dierkesmann R, Becker HD, Bolliger CT, Frank W, Freitag L, Gottschall DA, Häußinger K, Huber R, Kirsten D, Kreuzer A, Loddenkemper R, Macha HN, Mäder I, Mall W, Morr H, Nakhosteen J, Strausz J, Tekolf E, Wagner M, Wilkens H, Witt
C. Empfehlungen zur bronchoskopischen Behandlung tracheobronchialer Verschlüsse, Stenosen und muraler maligner Tumoren. Pneumologie 1998; 52: $243-248$

54 Personne C, Colchen A, Leroy M, Vourc'h G, Toty L. Indications and technique for endoscopic laser resections in bronchology. A critical analysis based upon 2.284 resections. J Thorac Cardiovasc Surg 1986; 91: 710-715

${ }^{55}$ Diaz-Jiménez JP, Martínez-Ballarín JE, Llunell A, Farrero E, Rodríguez A, Castro MJ. Efficacy and safety of photodynamic therapy versus Nd-YAG laser resection in NSCLC with airway obstruction. Eur Respir J 1999; 14: 800-805

${ }^{56}$ McCaughan Jr JS, Williams Jr TE, Bethel BH. Photodynamic therapy of endobronchial tumors. Lasers Surg Med 1986; 6: $336-345$

${ }^{57}$ Lam S, Cory P. Combined photodynamic therapy (PDT) using Photofrin and radiotherapy (XRT) versus radiotherapy alone in patients with inoperable distribution of non-small cell bronchogenic cancer. Proc SPIE 1991; 5: 20-28

${ }^{58}$ Dachowski LJ, DeLaney TF. Photodynamic therapy: The $\mathrm{NCl}$ experience and its nursing implications. Oncol Nurs Forum 1992; 19: $63-67$

${ }^{59}$ Cortese DA. Photodynamic Therapy for Early Stage Lung Cancer: A Treatment in Search of a Disease. Editorial. J Bronchol 1997; 4: 3-4

${ }^{60}$ Balchum OJ, Doiron DR. Photoradiation therapy of endobronchial lung cancer: Large obstructing tumors, non-obstructing tumors, early stage bronchial cancer lesions. Clin Chest Med 1985; 6: $255-275$

${ }^{61}$ Huber RM, Gamarra F, Hautmann H, Häußinger K, Wagner S, Castro M, Baumgartner R. 5-Aminolaevulinic Acid (ALA) for the Fluorescence Detection of Bronchial Tumors. Diagn Ther Endosc 1999; 5: 113 - 118

62 Huber RM, Gamarra F, Leberig A, Stepp H, Rick K, Häußinger K, Baumgartner R. Stellenwert der Fluoreszenzmethoden in der bronchologischen Diagnostik: Früherkennung des Bronchialkarzinoms möglich? Atemw Lungenkrkh 1995; 21: 558-561

${ }^{63}$ Kato $\mathrm{H}$. Photodynamic therapy for early stage central type of lung cancer - Editorial. Chest 1997; 72: 688-690

${ }^{64}$ Lam S. Photodynamic therapy in lung cancer. Semin Oncol 1994; 21: $15-19$

${ }^{65}$ LoCicero J, Metzdorff M, Almgren C. Photodynamic therapy in the palliation of late stage obstructing non-small cell lung cancer. Chest 1990; 98: $97-100$

${ }^{66}$ McCaughan JS. Photodynamic Therapy versus Nd-YAG laser treatment of endobronchial or esophageal malignancies; in Photodynamic Therapy and Biomedical Lasers. Proceedings of the International Conference on Photodynamic Therapy and Medical Laser Applications. Milan: 1992. 23-36

${ }^{67}$ Reynolds T. Using lasers and light-activated drugs, researches home in on early lung cancers. J Natl Cancer Inst 1998; 90: 411 418

\section{Dr. med. F. Stanzel}

Asklepios Fachkliniken München-Gauting

Pneumologische Klinik

Robert-Koch-Allee 2

82131 Gauting

E-mail: fstanzel@t-online.de 